\title{
Innere Medizin in den deutschsprachigen Ländern und in den USA
}

Wechselseitige Einflüsse und Wandel der Beziehungen von 1870 bis 1990

Arnd Schulte-Bockholt und Axel Bauer

\section{Summary}

Scientific relations in Internal Medicine between German-speaking countries and the United States were characterized by various stages during the last hundred and twenty years. From 1870 to the First World War German medical research and teaching as well as the competitive German university system were regarded as a model for US Medical Schools. After World War I, however, things changed as a result of American Medical Schools' reforms which had been implemented. On the other hand, financial equipment of German universities was decreasing due to inflation and unemployment. Furthermore, between 1933 and 1945, many excellent scientists and clinicians were forced to emigrate from Germany and Austria to the US because of persecution by the Nazis for political or "racial" reasons. After the end of World War II, scientific and technical leadership of American Internal Medicine remained unchallenged; today many European physicians complete their training in the biomedical sciences in the United States. Beginning in the 1980s, German-speaking internists increasingly publish the results of their research in American scientific journals.

\section{Zusammenfassung}

Die wissenschaftlichen Beziehungen auf dem Gebiet der Inneren Medizin zwischen den deutschsprachigen Ländern und den USA durchliefen

Dr. med. Arnd Schulte-Bockholt, Abteilung Gastroenterologie, Universitätsklinik Frankfurt, Theodor-Stern-Kai 7, Haus 11, D-60590 Frankfurt am Main

Prof. Dr. med. Axel Bauer, Institut für Geschichte der Medizin der Ruprecht-Karls-Universität, Im Neuenheimer Feld 368, D-69120 Heidelberg 
während der letzten 120 Jahre mehrere Stadien: In der Zeit von etwa 1870 bis zum Ersten Weltkrieg galten die deutsche Medizin in Forschung und Lehre sowie das kompetitive deutsche Hochschulsystem als vorbildlich für die Medical Schools der Vereinigten Staaten. Nach 1918 änderte sich das Bild jedoch infolge der qualitativen Konsolidierung der Medical Schools und aufgrund der sinkenden materiellen Standards an den deutschen Universitäten. Zwischen 1933 und 1945 kam es wegen der nationalsozialistischen Verfolgungen zusätzlich zu einem erheblichen personellen Transfer bedeutender Wissenschaftler und Kliniker in die USA. Nach dem Ende des Zweiten Weltkrieges blieb die wissenschaftliche und technische Führungsrolle der amerikanischen Internisten unangefochten; europäische Ärzte vervollständigen heute ihre biomedizinische Ausbildung in den Vereinigten Staaten. Im Laufe der 1980er Jahre nahm jedoch die Publikationsfrequenz deutschsprachiger Internisten und somit die Repräsentation ihrer Forschung in amerikanischen Fachzeitschriften deutlich zu.

\section{Einleitung}

Der Terminus Innere Medizin wurde erstmalig 1882 in den Verhandlungen des Wiesbadener Kongresses für Innere Medizin verwandt ${ }^{1}$.Zuvor hatte man das Gebiet der Inneren Medizin in Deutschland als Spezielle Pathologie und Therapie bezeichnet ${ }^{2}$. In den Vereinigten Staaten fand dieses Gebiet mit der Gründung der Association of American Physicians im Jahre 1886 seine erste Organisation $^{3}$. Die Termini Internist bzw. Internal Medicine wurden in den Vereinigten Staaten (nicht jedoch in England) als Lehnwort bzw. als Lehnübersetzung aus dem Deutschen übernommen. Ein wichtiger Grund hierfür war, dass von etwa 1870 bis zum Ersten Weltkrieg die amerikanische Innere Medizin bestimmende Impulse aus den deutschsprachigen Ländern erhielt. Es soll versucht werden, die wechselseitigen Einflüsse zwischen der Inneren

1 Die Autoren danken Herrn Prof. Konrad H. Soergel, M. D., vom Froedtert Memorial Lutheran Hospital, Medical College of Wisconsin, Milwaukee/ Wisconsin, für seine hilfreiche Unterstützung bei der Erarbeitung dieses Manuskripts.

2 Vgl. William B. Bean: Origin of the Term «Internal Medicine», N Engl J Med 306 (1982) 182-183. Zum folgenden siehe auch Erwin H. Ackerknecht: Die Medizin in den Vereinigten Staaten vor 1900, in: Ders., Geschichte der Medizin, 4. Auflage, Stuttgart 1979, 204-211. Simon Flexner und James Thomas Flexner: William Henry Welch und das heroische Zeitalter der amerikanischen Medizin, Stuttgart 1948. Francis R. Packard: History of Medicine in the United States, Bd. 1-2, New York 1931. Richard H. Shryock: Die Entwicklung der modernen Medizin, Stuttgart 1947. Henry E. Sigerist: Amerika und die Medizin, Leipzig 1933.

3 Vgl. Paul B. Beeson: One Hundred Years of American Internal Medicine. A View from the Inside, Ann Int Med 105 (1986) 436-444. 
Medizin des deutschen Sprachraums und der USA sowie den Wandel dieser Beziehungen von etwa 1870 bis in die Gegenwart darzustellen.

Wir verwenden den Begriff Innere Medizin in dem weiten Sinn, wie er in Deutschland im späten 19. Jahrhundert gebraucht wurde. Innere Medizin beruhte damals nicht zuletzt auf Experimenteller Physiologie und Biochemie, die als Forschungsgebiete auch in den Kliniken und Instituten für Innere Medizin betrieben wurden. Die Tradition der klinischen Grundlagenforschung durch Internisten besteht in begrenztem Rahmen in Deutschland auch heute noch weiter. Dagegen hat sich in den USA seit dem Zweiten Weltkrieg der Trend verstärkt, dass nicht mehr Kliniker, sondern Biowissenschaftler (Ph.D.) Grundlagenforschung betreiben, entweder als Mitglieder der Medizinischen Kliniken, in Biomedizinischen Departments von Universitäten oder in ausseruniversitären Institutionen wie den National Institutes of Health. Die folgende Darstellung umfasst sowohl die wechselseitigen Einflüsse in der Inneren Medizin im klinischen Sinne als auch in der internistischen Grundlagenforschung. Wir interpretieren den Begriff Deutschland dabei nicht nationalstaatlich, sondern sprach-kulturell. Aus amerikanischer Sicht umschliesst der Begriff der Deutschen Medizin bis zum Zweiten Weltkrieg auch Österreich-Ungarn, insbesondere Wien und Budapest, sowie die deutschsprachige Schweiz.

\section{Die Zeit von 1870 bis 1914}

Vor 1870 war die amerikanische Medizin überwiegend von den Universitäten in London, Edinburgh und Paris beeinflusst ${ }^{4}$. Dies geschah auf inidividueller Basis, durch Immigration englischer und französischer Ärzte und durch Studienaufenthalte von amerikanischen Medizinern in Europa. Als mittelbares Resultat von Wilhelm v. Humboldts (1767-1835) Universitätsreform (1810) waren unterdessen die modernen deutschen, forschungsorientierten Universitäten nach dem Modell der Berliner Friedrich-Wilhelm-Universität entstanden 5 . Forschung und Lehre wurden zu eindeutigen Schwerpunkten, geprägt durch das Konzept der Lehr- und Lernfreiheit. Dieses «Goldene Zeitalter» der deutschen Medizin war gekennzeichnet durch die Kombination naturwissenschaftlicher Grundlagenforschung - vor allem in Anatomie, Physiologie und Pathologischer Anatomie - mit einer Lehre, die über-

4 Vgl. Wilhelm Moll: History of American Medical Education, Brit J Med Educ 2 (1968) $173-181$.

5 Vgl. Laetitia Boehm: Wilhelm von Humboldt (1767-1835) and the University: Idea and Implementation, Mitteilungen der Alexander von Humboldt-Stiftung 46 (1985) 1-8. 
kommene medizinische Vorstellungen über Bord warf. Keine vergleichbare amerikanische Institution reichte zu Beginn des letzten Drittels des 19. Jahrhunderts an Universitäten wie Berlin, Leipzig, München, Heidelberg, Göttingen, Wien oder Budapest qualitativ heran. Fakultätsmitglieder der später führenden amerikanischen Medizinischen Hochschulen begannen ab etwa 1870, diese allgemein anerkannten deutschen Universitäten zu besuchen und ihren Aufbau zu studieren ${ }^{6}$. Ihr Ziel bestand darin, das deutsche System der Medizinerausbildung auf die Vereinigten Staaten zu übertragen. Sie hospitierten und arbeiteten jedoch vor allem in den Kliniken weltberühmter internistischer Ordinarien und nahmen dabei wenig Notiz von dem Werdegang der Medizinstudenten. Dieser bestand vorwiegend aus Vorlesungen und Krankenvorstellungen in grossen Auditorien, wenig direktem Kontakt mit den Professoren und einer Verschiebung der klinisch-praktischen Ausbildung auf die Zeit nach dem Staatsexamen. Folglich wurden diese Aspekte auch nicht in die sich entwickelnde Reform des amerikanischen Medizinstudiums übernommen?.

Die amerikanische Medizinerausbildung um das Jahr 1870 beruhte auf dem Lehrlingssystem in überwiegend privaten, kommerziell orientierten Schulen. Das Studienjahr war kurz, es dauerte von Oktober bis zum Frühjahr. Objektive Aufnahmekriterien gab es kaum. Fast jeder, der die Aufnahmegebühr bezahlte, wurde akzeptiert, die Prüfungen waren ziemlich lax ${ }^{8}$.

Zwischen 1870 und 1914 besuchten mindestens 10000 amerikanische Studenten, junge Ärzte wie auch erfahrene Praktiker deutsche, österreichischungarische und schweizerische Universitäten. Sie absolvierten ihr Medizinstudium, belegten kurze Privatkurse oder verbrachten gar mehrere Jahre in deutschen Forschungslaboratorien. Darunter waren vor 1890 auch Frauen, denen bis zu diesem Zeitpunkt in den USA das Medizinstudium weitgehend versagt war. Schweizer Universitäten liessen ab 1876 Frauen zum Studium zu; zwischen 1870 und 1910 studierten 120 Amerikaner in Zürich Medizin, davon 57 Frauen. In Deutschland wurden Frauen bis 1898 nicht zum Medizinstudium zugelassen. Die erste Amerikanerin, die 1871 ihr Studium in Zürich mit einer auf Deutsch geschriebenen Dissertation über die verschiedenen Formen des Kindbettfiebers abschloss, war Susan Dimock (1847-1875). Sie arbeitete von 1872 bis zu ihrem Tod bei einem Schiffsunglück 1875 als Assistenzärztin im New England Hospital in Boston und lei-

6 Siehe hierzu insbesondere Thomas N. Bonner: American Doctors and German Universities: A Chapter in International Intellectual Relations 1870-1914, University of Nebraska Press, Lincoln/London 1963 (1987).

7 Vgl. Thomas N. Bonner: The German Model of Training Physicians in the United States, 1870-1914: How Closely was it Followed?, Bulletin of the History of Medicine 64 (1990) 18-36. 8 Vgl. Anm. 6. 
tete dessen Schwesternschule. Eine der ersten Amerikanerinnen, die als Forschungsassistentin in einem deutschen Laboratorium arbeitete, war Alice Hamilton (1869-1970), eine Vorreiterin der arbeitsmedizinischen Forschung. Sie war in Leipzig und München, schliesslich im Pathologischen Institut in Frankfurt am Main unter Carl Weigert (1845-1904) tätig.

Die grosse Zahl von Ärzten, die klinisch und naturwissenschaftlich im deutschen Sprachraum ausgebildet wurde, beeinflusste Forschung, Lehre und Praxis der amerikanischen Innneren Medizin grundlegend. Rund 50\% der führenden Ärzte jener Zeit haben zumindest einen Teil ihrer Ausbildung in Deutschland, Österreich-Ungarn oder der Schweiz erworben. Wien und Berlin waren bekannt für Spezialkurse in umgrenzten Wissensgebieten oder Methoden, und im ausgehenden 19. Jahrhundert wurden diese Kurse oft sogar auf Englisch abgehalten. Eine relativ kleine Gruppe amerikanischer Ärzte kam nach Europa, um eine längere biomedizinische Ausbildung in pathologischen, physiologischen und biochemischen Instituten in Leipzig, Strassburg, Heidelberg oder Breslau zu erwerben. Aus diesen Nachwuchswissenschaftlern rekrutierten sich später die Professoren, welche die Medizinischen Fakultäten der Universitäten von Johns Hopkins in Baltimore, Harvard, Michigan und Yale in Anlehnung an das deutsche Universitätsmodell aufbauten und damit den Ruf der USA in der wissenschaftlichen Medizin begründeten. Die deutsche Medizin wurde zum Modell für klinische wie wissenschaftliche Standards, medizinische Ausbildung, Medizintechnik, Krankenhausbau und Medizinjournalismus. Wissenschaftliche Zeitschriften wie die Archives of Internal Medicine entstanden nach dem deutschen Vorbild ${ }^{9}$. Auch der Journal Club als Instrument der Lehre und der kritischen Diskussion wurde von Deutschland-Heimkehrern in das amerikanische Ausbildungssystem eingeführt. Diese Institution wurde nach dem Zweiten Weltkrieg von deutschen Forschern, welche die USA besucht hatten, nach Deutschland reimportiert ${ }^{10}$.

Der Strom amerikanischer Studenten an deutschsprachige Universitäten war so gross, dass spezielle Führer für amerikanische Medizinstudenten in Europa veröffentlicht wurden. Darin wurde Wien für die klinische und die Universitäten des Deutschen Reichs für die Laboratoriumsausbildung empfohlen. Sie enthielten auch praktische Tips, so etwa die Empfehlung, dass sich der Studierende einen Studentenausweis besorgen sollte; dessen Hauptvor-

9 Vgl. W. Bruce Fye: The Literature of Internal Medicine, in: Russell C. Maulitz und Diana E. Long (Hg.), Grand Rounds. One Hundred Years of Internal Medicine, University of Pennsylvania Press 1988, 55-84.

10 Vgl. Mark Linzer: The Journal Club and Medical Education: Over One Hundred Years of Unrecorded History, Postgraduate Med J 63 (1987) 475-478. 
teil sei, dass man im Falle eines Konfliktes mit der Gendarmerie in das Universitätsgefängnis und nicht das Zivilgefängnis eingewiesen werde ${ }^{11}$. Es entstanden Vereine amerikanischer Mediziner an deutschsprachigen Universitäten, zum Beispiel eine American Medical Association (nicht zu verwechseln mit der gleichnamigen amerikanischen Ärzteorganisation AMA), die 1904 in Wien gegründet wurde und bis 1939 bestand. Aufgabe der Vereine war es, Vorlesungsverzeichnisse herauszugeben, Professoren zu Vorlesungen einzuladen sowie sozialer Treffpunkt zu sein.

In den 1870er Jahren begannen einzelne Universitätsreformer, aus Europa eingewanderte und an deutschsprachigen Universitäten ausgebildete Ärzte, eine Reform der amerikanischen Medizinerausbildung zu empfehlen ${ }^{12}$. In Berlin, Wien oder Heidelberg hatten sie ein Ausbildungssystem kennengelernt, das durch Weiterbildung nach dem Studienabschluss und durch Professoren gekennzeichnet war, die vollamtlich lehren und forschen konnten, da sie nicht darauf angewiesen waren, eine Privatpraxis zur Sicherung ihres Lebensunterhaltes zu betreiben. Sie kannten gut ausgestattete Laboratorien und eine Atmosphäre, die individuelle Forschungsinteressen entwickelte und förderte. Johns Hopkins (1795-1873), ein wohlhabender Kaufmann aus Baltimore, beauftragte John Shaw Billings (1838-1913), die Medizinische Fakultät der nach ihm benannten und von ihm finanzierten Universität zu planen. Obwohl die beiden nie im Ausland studiert hatten, entwarfen sie das Modell einer Universität und Medizinischen Fakultät, das sich stark an deutsche Einflüsse anlehnte. Billings als Planer und Daniel C. Gilman (1831-1908) als Präsident der neugegründeten Universität reisten nach Deutschland, um Organisation und Ausstattung der Universitäten zu studieren und sich mit deutschen Professoren über Auswahl und Qualifikation der zu ernennenden Fakultätsmitglieder zu beraten. Es begaben sich auch die Präsidenten der Harvard University und der University of Michigan, James Clark White (1833-1916) von Cornell sowie George A. Blumer (1858-1940), Dekan an der Yale University, an deutschsprachige Universitäten, um dieses System in ihre Reformen einfliessen zu lassen. So rekrutierten die Leiter und Lehrstuhlinhaber der Medizinischen Fakultäten der Universitäten von Johns Hopkins, Harvard, Yale und Michigan viele in Deutschland ausgebildete Ärzte.

11 Siehe bei Richard Warren: Reflections on Nineteenth Century German Medicine, Am J Surg 135 (1978) 461-468.

12 Vgl. Russell C. Maulitz: Grand Rounds: An Introduction to the History of Internal Medicine, in: Russell C. Maulitz und Diana E. Long (Hg.), Grand Rounds. One Hundred Years of Internal Medicine, University of Pennsylvania Press 1988, 3-14. 
Der Einfluss der deutschen Medizin wird am Beispiel der Johns Hopkins University deutlich ${ }^{13}$ : Daniel C. Gilman und John Shaw Billings wählten junge, in Deutschland ausgebildete Professoren für die neu zu besetzenden Lehrstühle aus. John J. Abel (1857-1938), Howard Kelly (1858-1943), Franklin P. Mall (1862-1917), William S. Halsted (1852-1922), William H. Welch (1850-1934) und William Osler (1849-1919), klinisch und in den Naturwissenschaften an deutschen Universitäten ausgebildet, waren davon begeistert, wie in Deutschland Medizin gelehrt und praktiziert wurde. Osler beschrieb dies mit folgenden Worten: «Es ist mein Ehrgeiz, eine grosse Klinik nach deutschem Vorbild aufzubauen, nicht nach dem Vorbild Englands, sondern in Anlehnung an das deutsche Modell, das Deutschland zum führenden Platz wissenschaftlicher Medizin machte» ${ }^{14}$. Auch mehrere prominente Fakultätsmitglieder der Harvard Medical School verbrachten bis 1914 Studienaufenthalte an deutschen Universitäten; darunter waren Milton J. Rosenau (1869-1946), James Clark White (1833-1916), Henry Pickering Bowditch (1840-1911), Harold C. Ernst (1856-1922), Charles S. Minot (1852-1914), Thomas Dwight (1843-1911), William T. Councilman (1854-1933), Henry W. Williams (1821-1895), Frederick I. Knight (1841-1909) und John C. Warren (1842-1927). Das Kollegium der Medizinischen Fakultät der Yale Medical School war ebenfalls grossenteils in Deutschland ausgebildet worden, so William H. Carmalt (1836-1929), Graham Lusk (1866-1932), Ross G. Harrison (1870-1959), George A. Blumer und Russell H. Chittenden (1856-1943). Letzterer baute das nach deutschem Modell errichtete Physiologische Institut in Yale auf. Er hatte seine wissenschaftliche Ausbildung im Physiologischen Institut der Universität Heidelberg bei Willy Kühne (1837-1900) erworben.

Eine andere frühzeitig modernisierte Universität war die University of Michigan, wo an der Medizinischen Fakultät Victor C. Vaughan (1851-1929) (Hygiene und Biochemie), John J. Abe ${ }^{15}$ und Arthur R. Cushny (1866-1926)

$13 \mathrm{Vgl}$. Abner McGehee Harvey et al.: A Model of Its Kind. Vol. I. A Centennial History of Medicine at Johns Hopkins. Vol. II. A Pictorial History of Medicine at Johns Hopkins, The Johns Hopkins University Press, Baltimore/London 1989.

14 Vgl. Anm. 6.

15 John Jacob Abel stammte aus der Nähe von Cleveland, Ohio. Er studierte von 1876 bis 1883 an der University of Michigan, arbeitete anschliessend für ein Jahr im Laboratorium von Newell Martin in Baltimore und hielt sich sodann von 1884 bis 1890 in Deutschland auf - länger als jeder andere bedeutende amerikanische Naturwissenschaftler seiner Generation. Seine Stationen waren Leipzig (Carl Ludwig), Strassburg (Promotion 1888), Würzburg, Heidelberg, Wien, Bern und erneut Leipzig. 1891 kehrte er zunächst als Dozent für «Materia Medica und Therapie» an die University of Michigan zurück, erhielt jedoch 1893 den Lehrstuhl für Pharmakologie an der Johns Hopkins University in Baltimore, den er bis zu seiner Emeritierung 1932 innehatte. Vgl. Charles E. Rosenberg: Abel, John Jacob, in: Charles Coulston Gillispie (Hg.), Dictionary of Scientific Biography, Bd. 1, New York 1970, 9-12. 
(Pharmakologie), Paul C. Freer (1862-1912) (Chemie), William Howell (1860-1945) und Warren P. Lombard (1855-1939) (Physiologie) sowie Frederick Novy (1864-1957) (Bakteriologie) in Deutschland ausgebildet worden waren. Nach kontinentalem Modell gestaltete Fakultäten wie Johns Hopkins, Harvard, Cornell, Michigan und Yale waren jedoch eher Ausnahmen; die Mehrheit der Medical Schools erreichte am Ende des 19. Jahrhunderts noch nicht die europäischen Standards in Forschung und Lehre.

Im Auftrag der Carnegie Foundation for the Advancement of Teaching erstellte der aus Louisville in Kentucky stammende Ausbildungsexperte Abraham Flexner (1866-1959), ein jüngerer Bruder des an der Johns Hopkins University lehrenden Pathologen und Bakteriologen Simon Flexner (1863-1946), in den Jahren 1910 bzw. 1912 je einen Bericht über das Medizinstudium in den USA und Kanada bzw. in Europa ${ }^{16}$. Er verglich die 148 amerikanischen Medical Schools mit europäischen Medizinischen Fakultäten. Der Flexner Report kam zu dem Schluss, dass das Niveau der amerikanischen medizinischen Hochschulausbildung sehr unterschiedlich, aber mit Ausnahme der wenigen Reformuniversitäten generell unzureichend war. Als Folge dieses Berichtes setzte sich die an Lehre und Forschung orientierte und an eine Universität gebundene Medical School durch. Diese Anpassung blieb jedoch auf den akademischen Bereich beschränkt. Viele Medical Schools und Lehrkrankenhäuser blieben in den Händen gemeinnütziger Gremien oder prominenter Bürger. Die Akkreditierung der Lehranstalten sowie das medizinische Staatsexamen wurden von einflussreichen Standesorganisationen geregelt und nicht vom Staat kontrolliert.

Zwei Beispiele herausragender deutscher Lehrer und ihrer amerikanischer Schüler seien hier kurz dargestellt: Carl Ludwig (1816-1895) war der führende Physiologe seiner Zeit ${ }^{17}$. Sein Physiologisches Institut in Leipzig war weltweit Vorbild für physiologische Forschungs- und Lehrinstitute. Bis zum heutigen Tag lässt sich der «Stammbaum» vieler Physiologen auf Carl Ludwig zurückführen. Seine beiden bekanntesten amerikanischen Schüler waren William H. Welch, Professor für Pathologie und später Dekan an der Johns Hopkins University sowie Henry Pickering Bowditch. Welch und Bow-

16 Abraham Flexner: Medical Education in the United States and Canada: A Report to the Carnegie Foundation for the Advancement of Teaching, Bulletin Number 4, New York 1910. Abraham Flexner: Medical Education in Europe: A Report to the Carnegie Foundation for the Advancement of Teaching, Bulletin Number 6, New York 1912. Zu Leben und Werk Abraham Flexners vgl. auch Abner McGehee Harvey et al.: A Model of Its Kind. Vol. I. A Centennial History of Medicine at Johns Hopkins, The Johns Hopkins University Press, Baltimore/London 1989, 50 und passim.

17 Siehe bei Chandler McC Brooks und Horst Seller: Early and Late Contributions to our Knowledge of the Autonomic Nervous System and its Control Made by German Scientists, J Autonomic Nervous System 3 (1981) 105-119. 
ditch beeinflussten ihrerseits die Entstehung des Flexner-Reports, der zur Grundlage der Reform des amerikanischen medizinischen Ausbildungssystems wurde. William H.Welch besuchte Carl Ludwigs Institut mehrmals. Für die führende Rolle Ludwigs in der Physiologie seiner Zeit spricht auch die Tatsache, dass zur selben Zeit (1885-1886) im Leipziger Institut sowohl Welch, einer der Väter der modernen Pathologie und Hygiene in Amerika, als auch Iwan Petrowitch Pawlow (1849-1936), der Begründer der modernen russischen Physiologie, arbeiten ${ }^{18}$. Bowditch erlernte in Ludwigs Laboratorium den Gebrauch des Kymographen, einer russgefärbten rotierenden Trommel zur graphischen Aufzeichnung physiologischer Phänomene. Bowditch wurde später auf den Lehrstuhl für Physiologie an der Harvard Medical School berufen. Er lehnte dies jedoch zunächst ab, um seine Studien bei Ludwig fortsetzen zu können. 1871 nahm er schliesslich den zweiten Ruf an. Mit Laboreinrichtungen, die er in Leipzig erworben hatte, eröffnete er das erste Physiologische Institut in den USA. Innerhalb weniger Jahre entstanden weitere Physiologische Institute, zuerst in Michigan unter Warren P. Lombard und an der Johns Hopkins University unter Newell Martin (1848-1896), beide Studenten bzw. Schüler Carl Ludwigs ${ }^{19} .1870$ wurde in der Zeitschrift Nature ein Artikel veröffentlicht, der Aufbau und Organisation des Physiologischen Instituts in Leipzig beschrieb ${ }^{20}$.

Weitere bekannte amerikanische Ludwig-Schüler waren George A. Blumer, später Dekan an der Yale Medical School, Henry Sewall (1855-1936), Warren P. Lombard, Frederick S. Lee (1859-1939), Charles S. Minot, John J. Abel und Franklin P. Mall. Viele von Ludwigs Schülern wurden ebenfalls zu Lehrern amerikanischer Forscher. So bildete der später als Ordinarius in Bern lehrende Physiologe Hugo von Kronecker (1839-1914) schon in Berlin und Königsberg Samuel J. Meltzer (1851-1920) aus, einen Begründer der amerikanischen Gastroenterologie und Leiter der Pharmakologie und Physiologie am Rockefeller Institute ${ }^{21}$.

$18 \mathrm{Vgl}$.W. Bruce Fye:The Origin of the Full-Time Faculty System. Implications for Clinical Research, JAMA 265 (1991) 1555-1562. Pawlow begab sich Anfang Juni 1884 zu einem zweijährigen Studienaufenthalt in die Physiologischen Institute von Rudolf Heidenhain (1834-1897) nach Breslau und von Carl Ludwig nach Leipzig. Vgl. hierzu Lothar Pickenhain: Iwan Petrowitsch Pawlow (1849-1936), in: Dietrich von Engelhardt und Fritz Hartmann (Hg.), Klassiker der Medizin II, München 1991, 284-298, hier S. 287. Zu William H. Welch, der in Leipzig auch bei dem Pathologen Julius Cohnheim studierte, vgl. unten Anm. 24.

19 Vgl. Hebbel E. Hoff: Medical Progress a Century ago: A New Medical Curriculum in America, Connecticut Medicine 36 (1972) 57-59.

20 Vgl. Hebbel E. Hoff: Medical Progress a Century ago: The Physiology Laboratory Comes to America. Part II, Connecticut Medicine 36 (1972) 109-115.

21 Joseph B. Kirsner: The Development of American Gastroenterology, Raven Press, New York 1990 sowie ders.: One Hundred Years of American Gastroenterology, in: Russell C. Maulitz und Diana E. Long (Hg.), Grand Rounds: One Hundred Years of Internal Medicine, University of Pennsylvania Press 1988, 117-157, hier S. 125. 
Am Beispiel des Münchener Ordinarius für Innere Medizin, Friedrich von Müller (1858-1941), soll der Einfluss der deutschen Klinik auf die Vereinigten Staaten erläutert werden ${ }^{22}$.Zwischen 1900 und 1914 studierten 165 Amerikaner in München. Aufgrund dieser Kontakte erlernte von Müller Englisch und publizierte schliesslich auch in amerikanischen Zeitschriften. Abraham Flexner war ein häufiger Besucher in von Müllers Klinik. Graham Lusk, ein Schüler von Müller, schrieb ${ }^{23}$ : «Als medizinischer Lehrer ist Friedrich von Müller der beste in der Welt, und es gibt niemanden in Amerika, der qualifiziert wäre, eine Klinik wie die Friedrich von Müllers zu leiten». Zu seinen Schülern gehörten weiter auch Lewellys F. Barker (1867-1943), der Nachfolger William Oslers am Johns Hopkins Hospital, und dessen Schüler Rufus Cole (1872-1966), der auf Anraten Barkers bei von Müller arbeitete, bevor er die Leitung des Rockefeller Institute Hospitals übernahm. Ein begeisterter Hörer der Vorlesungen Friedrich von Müllers war im Sommersemester 1914 übrigens auch der 23jährige Medizinstudent Henry E. Sigerist (1891-1957), der später zwischen 1925 und 1932 als Nachfolger Karl Sudhoffs (1853-1938) Direktor des Instituts für Geschichte der Medizin an der Universität Leipzig werden sollte und der anschliessend von 1932 bis 1947 das Institut für Geschichte der Medizin an der Johns Hopkins University in Baltimore leitete, das er von dem damals 82jährigen William H. («Popsie») Welch übernahm ${ }^{24}$. Sigerist charakterisierte Friedrich von Müller im Jahre 1946 rückblickend so: «... ein echter Gentleman, ein hervorragender Arzt und Naturwissenschaftler und ein hochkultivierter Mensch; der typische deutsche Professor im besten Sinne des Wortes ... Von Müllers Unterricht war so eindrucksvoll, dass ich mich bis auf den heutigen Tag an jeden einzelnen Fall erinnere, den er uns im Laufe einer Vorlesung vorführte, ja sogar die Gesichter der Patienten noch vor mir sehe. Seine Vorlesungen waren immer überfüllt, und jeden Morgen setzte ein regelrechtes Rennen auf Sitzplätze ein $»^{25}$. Zusammenfassend lässt sich sagen, dass Grundlagenforscher wie Carl Ludwig, Felix Hoppe-Seyler (1825-1895), Wilhelm His (1831-1904), Friedrich Daniel von Recklinghausen (1833-1910), Wilhelm von Waldeyer-Hartz

22 Siehe Thomas N. Bonner: Friedrich von Müller of Munich and the Growth of Clinical Science in America, 1902-1914, Journal of the History of Medicine and Allied Sciences 45 (1990) $556-569$.

$23 \mathrm{Vgl}$. Anm. 22.

24 Vgl. dazu Achim Thom und Karl-Heinz Karbe: Henry Ernest Sigerist (1891-1957). Begründer einer modernen Sozialgeschichte der Medizin. Ausgewählte Texte (= Sudhoffs Klassiker der Medizin, N.F.1), Leipzig 1981. Über William H. Welch siehe Gert H. Brieger:Welch, William Henry, in: Charles Coulston Gillispie (Hg.), Dictionary of Scientific Biography, Bd. 14, New York 1976, 248-250.

25 Henry E. Sigerist: Autobiographische Schriften, ausgewählt von Nora Sigerist Beeson, ins Deutsche übertragen von Alice Meyer, Stuttgart 1970, 60-61. 
(1836-1921), Oswald Schmiedeberg (1838-1921) und Julius Cohnheim (1839-1884) sowie Kliniker wie Friedrich Theodor Frerichs (1819-1885), Bernhard Naunyn (1839-1925) und Friedrich von Müller einen bestimmenden Einfluss auf die Entwicklung der wissenschaftlichen Medizin der Vereinigten Staaten ausübten. Zu ihren Studenten zählten die Begründer der amerikanischen Inneren Medizin, der Physiologie, der Biochemie, der Pharmakologie und der Pathologie.

Um die Wende zum 20. Jahrhundert begann die amerikanische Medizin jedoch auch ihrerseits auf die deutsche Medizin Einfluss zu nehmen. Zwischen 1904 und 1908 reisten Friedrich von Müller, Paul Ehrlich (1854-1915) und Robert Koch (1843-1910) zu Studien in die Vereinigten Staaten. Friedrich von Müller lobte 1907 - wenn auch mit unüberhörbarer Ironie - insbesondere die Einrichtungen amerikanischer Lehrlaboratorien: «Wenn wir in den deutschen Kursen Experimente an der Maus ausführen, so nehmen die Amerikaner dafür zum gleichen Zwecke einen Elefanten». Über den Stand der amerikanischen Medizinliteratur verkündete er: «Abgesehen davon, dass die Veröffentlichungen in Englisch gehalten sind, könnten sie auch in Deutsch geschrieben sein». Von Müller war erstaunt über das medizinische Wissen der Amerikaner, schrieb dies jedoch noch immer selbstbewusst dem deutschen Einfluss und den Studien amerikanischer Ärzte in Deutschland zu. Ähnlich äusserte sich Robert Koch, der 1908 nach einer amerikanischen Studienreise bemerkte: «Die meisten wissenschaftlichen Entwicklungen auf dem Gebiet der Bakteriologie wurden in Deutschland gemacht, aber ich muss zugeben, dass hier in Amerika und besonders in New York sie uns in der praktischen Anwendung weit voraus sind $»^{26}$.

\section{Die Zeit von 1914 bis 1945}

Der Erste Weltkrieg markierte das Ende einer Ära. In Deutschland lag die Volkswirtschaft 1918 am Boden, die Universitäten waren bankrott. Im Gegensatz zum europäischen Kontinent gingen die USA wirtschaftlich gestärkt aus dem Krieg hervor. Der während des Krieges aufgekommene Chauvinismus einerseits sowie die nahezu abgeschlossenen Veränderungen im amerikanischen medizinischen Ausbildungssystem andererseits führten nun dazu, dass amerikanische Studenten sowie Ärzte nach Abschluss ihres Studiums zunehmend einheimische Universitäten für ihre Aus- und Weiterbildung

26 Vgl. Anm. 6.

27 Edward Mornan: Baltimore, MD, Persönliche Mitteilung vom 1. März 1993. 
wählten. Zwar reisten weiterhin junge Ärzte nach Deutschland und Österreich, in der Hoffnung, an die Zeit vor dem Krieg anknüpfen zu können. Sie erkannten aber bald, dass sich die deutsche Medizinerausbildung qualitativ verschlechtert hatte, und sie sahen jetzt auch die vergleichsweise negativen Aspekte des deutschen Systems. Dazu zählten die geringe Beachtung der Patienten, das fehlende Engagement für nicht an Forschung interessierte Studenten und ein Mangel an Orginalität der klinischen Forschung.

Die Gründe, aus denen Amerikaner eine Ausbildung in Europa anstrebten, änderten sich. Es begaben sich plötzlich solche Studenten an deutschsprachige Universitäten, welche die Aufnahme in ein amerikanisches Ausbildungsprogramm nicht erreicht hatten. Zu Beginn der 1930er Jahre wurde die Anzahl solcher Studenten in Deutschland, Österreich und der Schweiz auf insgesamt 500 pro Jahr geschätzt. In den 1920er und frühen 1930er Jahren kamen mehrere hundert amerikanische Juden nach Deutschland, um dort Medizin zu studieren ${ }^{27}$. Der Grund hierfür war ein inoffizieller Numerus clausus, der Juden den Zugang zu mehreren amerikanischen Universitäten erschwerte ${ }^{28}$. Einige dieser amerikanischen Medizinstudenten kehrten erst 1935/36 in die USA zurück. Aufgrund der grossen Zahl amerikanischer Medizinstudenten, die in Deutschland studierten, plante die American Medical Association (AMA) Anfang der 1930er Jahre, die Zulassungsmöglichkeiten für diejenigen amerikanischen Mediziner einzuschränken, die im Ausland studiert hatten. Dies betraf vor allem jüdische Mediziner.

Parallel zu dieser Entwicklung verstärkte sich allerdings eine Gegenströmung, die bereits um die Jahrhundertwende begonnen hatte. Deutsche Ärzte bereisten die USA, um Fortschritte, speziell der Inneren Medizin und Chirurgie, zu beobachten und zu studieren. Die Richtung des Forschungstransfers hatte sich also seit den Tagen des Besuchs von Welch in Deutschland weitgehend umgekehrt. Damit hatte sich verwirklicht, was Nicholas Senn (1844-1908) schon 1902 vorhergesagt hatte ${ }^{29}$. Senn war ein amerikanischer Chirurg, der 1877 unter Johann Nepomuk von Nussbaum (1829-1890) in München dessen experimentelle chirurgische Forschungsmethoden studiert hatte. Nach der Rückkehr in die USA wurde er durch grundlegende Arbeiten zur Herzkatheterisierung bei Tieren sowie durch die Digitalistherapie bekannt. Senn sagte damals: «In weniger als 25 Jahren werden die USA das Mekka sein, zu dem Medizinstudenten aus aller Welt strömen werden». Werner Forssmann (1904-1979), Chirurgischer Assistenzarzt in Eberswalde,

28 Vgl. Kathleen M. Pearle: Ärzteemigration nach 1933 in die USA: Der Fall New York, $M e$ dizinhistorisches Journal 19 (1984) 112-137.

29 Vgl. Paul A. Carlson: Three Contributors to Cardiac Catherization, J Thorac Cardiovasc Surg 79 (1980) 782-788. 
führte 1929 die erste Herzkatheterisierung im Selbstversuch durch. Er konnte sein wegweisendes Experiment jedoch gegen den Widerstand des führenden Thoraxchirurgen Ferdinand Sauerbruch (1875-1951) in Berlin nicht fortsetzen. Sauerbruch tat Forssmann mit der Bemerkung ab, er betreibe «eine Klinik und keinen Zirkus» ${ }^{30}$. Die Methode wurde von den Kardiologen André F. Cournaud (1895-1988) und Dickinson W. Richards (1895-1973) während des Zweiten Weltkrieges zur klinischen Routine weiterentwickelt. Forssmann wurde erst in den Nachkriegsjahren in Deutschland «wiederentdeckt», so dass dieses Trio der Klinischen Kardiologie im Jahre 1956 den Nobelpreis erhielt.

In den 1920er und 1930er Jahren traten die Vereinigten Staaten von Amerika das wissenschaftliche Erbe der deutschen Medizin an. Die Gründe dafür waren zum einen der Niedergang der deutschen Medizinerausbildung, der sich mit der Machtübernahme der Nationalsozialisten 1933 beschleunigte, und zum anderen die abgeschlossene Reform des amerikanischen Medizinstudiums. Die Machtergreifung der Nationalsozialisten 1933 führte bald zu einer qualitativen Verarmung der deutschen - und ab 1938 - auch der österreichischen Universitäten und Medizinischen Fakultäten ${ }^{31}$. Mehrere bedeutende Internisten jener Zeit waren Juden ${ }^{32}$, darunter zum Beispiel der Freiburger Ordinarius für Innere Medizin, Siegfried Thannhauser (1885-1962) (33. $^{33}$. Während 1933 zunächst nur 11,4\% der aufgrund nationalsozialistischer Verfolgung emigrierenden Ärzte in die USA auswanderten, stieg dieser Prozentsatz bereits 1934 auf 32,7\% und lag ab 1936 in der Regel über 50\% ${ }^{34}$. Unter den in die USA immigrierten Ärzten waren Psychiater und Internisten am stärksten vertreten, sie machten 19,3\% bzw. 12,7\% aller eingewanderten Ärzte aus ${ }^{35}$. Die Zahl der aus Deutschland immigrierten Mediziner war so

30 Siehe Anm. 29.

$31 \mathrm{Vgl}$. hingegen Mitchell G. Ash, Wissenschaftswandel in Zeiten politischer Umwälzungen Entwicklungen, Verwicklungen, Abwicklungen (Karl-Sudhoff-Gedächtnisvortrag), Nachrichtenblatt der Deutschen Gesellschaft für Geschichte der Medizin, Naturwissenschaft und Technik e.V. 43 (1993) 133-147, hier S. 140-141. Ash bestreitet die These eines linearen Wissenstransfers durch die emigrierten Forscher im Sinn einer simplen Gewinn- und Verlustrechnung. Man müsse vielmehr fragen, «ob oder wie solche erzwungene Karriere oder geistigen Brüche vielleicht ironischerweise sogar innovationsfördernd wirkten».

32 Siehe Hans-Peter Kröner: Die Emigration deutschsprachiger Mediziner im Nationalsozialismus, Berichte zur Wissenschaftsgeschichte, Organ der Gesellschaft für Wissenschaftsgeschichte, Sonderheft, Weinheim 1989, 29 (Anmerkung 32).

$33 \mathrm{Vgl}$. Anm. 32.

34 Siehe Hans-Peter Kröner: Die Emigration deutschsprachiger Mediziner im Nationalsozialismus, Berichte zur Wissenschaftsgeschichte, Organ der Gesellschaft für Wissenschaftsgeschichte, Sonderheft, Weinheim 1989, 39 (Tabelle 7).

35 Siehe Hans-Peter Kröner: Die Emigration deutschsprachiger Mediziner im Nationalsozialismus, Berichte zur Wissenschaftsgeschichte, Organ der Gesellschaft für Wissenschaftsgeschichte, Sonderheft, Weinheim 1989, 44 (Tabelle 19). 
gross, dass in New York eine deutsche wissenschaftliche Gesellschaft, die Virchow Society gegründet wurde, die später in der New York Academy of Medicine aufging. Im folgenden wollen wir anhand einiger Einzelschicksale den Einfluss dieser Immigrationswelle auf die amerikanische Innere Medizin belegen.

Joseph H.Pratt (1872-1956), der Leiter der Pratt Clinic, dem späteren New England Medical Center, rekrutierte vor und nach der Machtübernahme der Nationalsozialisten gezielt deutsche Ärzte für seine Klinik ${ }^{36}$. Darunter waren der Frankfurter Biochemiker Gerhard Schmidt (1901-1981), ein Pionier der Nukleinsäuren- und Hirnlipidforschung ${ }^{37}$, und der Freiburger Internist Siegfried Thannhauser. Thannhauser, dessen Eltern zum Christentum konvertierten, war sich nach Aussagen eines ehemaligen Mitarbeiters ${ }^{38}$ kaum bewusst, dass seine Vorfahren Juden gewesen waren. Gleichwohl verlor er 1933 seinen Freiburger Lehrstuhl. Thannhauser war ein biochemisch orientierter Internist, hatte ein Buch über Stoffwechselerkrankungen geschrieben und wurde nach Übersiedlung in die Vereinigten Staaten eine weltweit anerkannte Autorität für Fettstoffwechselerkrankungen ${ }^{39}$.

Franz Maximilian Grodel (1881-1951) war in Frankfurt am Main Kardiologe und Radiologe gewesen ${ }^{40}$. Er entwickelte einen schnelltransportierenden Platten-Sereographen, den Vorgänger der direkten Röntgenkinomatographie, und er beschrieb das Konzept der unipolaren Brustwandableitungen. Er war Mitbegründer der Deutschen Gesellschaft für Kreislaufforschung und plante zusammen mit seinem Patienten William G. Kerckhoff (18561929), einem reichen Deutsch-Amerikaner aus Los Angeles, seit 1927/28 das Kerckhoff-Forschungsinstitut mit einer Klinik für Herz- und Kreislauferkrankungen in Bad Nauheim. Nach dem Tod Kerckhoffs finanzierte dessen Witwe Louise den Bau weiter, so dass das Institut in den Jahren 1929 bis 1931 fertiggestellt werden konnte. 1933 wurde Franz Groedel mit der Begründung, er sei «Nichtarier», die Venia legendi aberkannt, und er musste Deutschland verlassen ${ }^{41}$. In seiner neuen Heimat, den USA, wurde er aktiv

36 Herbert Black: Doctor and Teacher, Hospital Chief. Dr. Samuel Proger and the New England Medical Center, Globe Pequot Press, Chester/ Connecticut 1982.

37 Vgl. Peter Kröner: Vor fünfzig Jahren. Die Emigration deutschsprachiger Wissenschaftler 1933-1939, Gesellschaft für Wissenschaftsgeschichte Münster 1983, 47.

38 Harold Reinstein: Boston, MA, Persönliche Mitteilung vom 22. März 1993.

39 Samuel Proger: Eulogy Siegfried Josef Thannhauser, M.D. Gehalten im Tufts New England Medical Center am 20. Dezember 1962.

40 Vgl. M. Schlepper: Franz Maximilian Groedel - ein deutsches Schicksal von internationaler kardiologischer Bedeutung, Ztschr Kardiol 77 (1988) Suppl. 5, 155-177.

41 Über seine Erlebnisse als «halbjüdischer» Arzt im Kerckhoff-Institut während des Jahres 1944 berichtet in seiner Autobiographie der Giessener Internist Werner Schmidt: Leben an Grenzen. Autobiographischer Bericht eines Mediziners aus dunkler Zeit. Suhrkamp Taschenbuch Verlag, Frankfurt am Main 1993, 139-155. 
in der New Yorker Rudolph-Virchow-Society und der New York Cardiological Society. 1949 gründete er zusammen mit wenigen Freunden das American College of Cardiology, zu dessen erstem Präsidenten er gewählt wurde. Aus Kindheitstagen kannte er den späteren Präsidenten Franklin D. Roosevelt (1882-1945), mit dem er 1891 während eines Kuraufenthaltes der Familie Roosevelt in Bad Nauheim zur Schule gegangen war. Groedel wurde auf Veranlassung Roosevelts in die Saratoga Springs Commission gewählt, um den amerikanischen Rehabilitations- und Badebetrieb zu reorganisieren. Nach dem Zweiten Weltkrieg wurde Groedel gebeten, das Kerckhoff-Institut der Max-Planck-Gesellschaft in Bad Nauheim wieder zu übernehmen. Groedel blieb zwar von New York aus formal weiterhin Institutsdirektor, aber die wissenschaftliche Leitung übernahm 1951 der in Frankfurt am Main 1935 habilitierte Physiologe Rudolf Thauer (1906-1986), der seine Bestallung als Stellvertretender Direktor des Bad Nauheimer Max-Planck-Instituts für Physiologische und Klinische Forschung noch persönlich bei Groedel in New York entgegennahm ${ }^{42}$. Groedel sandte Container mit Lebensmitteln, Kleidung sowie Laborausrüstungen aus den USA nach Bad Nauheim. Seit 1945 wird die Franz-Groedel-Medaille als Auszeichnung für Kardiologie durch die Yale University verliehen. Franz Groedel starb 1951 durch einen Unfall in New York, mitten in den Vorbereitungen zur ersten Jahrestagung des American College of Cardiology ${ }^{43}$.

Der 1938 in die USA emigrierte Kölner Kreislaufphysiologe und Biochemiker Bruno Kisch (1890-1966) ${ }^{44}$ schrieb über den Einfluss der Deutschen auf die amerikanische Kardiologie: «Das American College of Cardiology, dies jüngste Kind der Deutschen Gesellschaft für Kreislaufforschung, ist sich seiner Vaterschaft kaum je bewusst, spielt jedoch heute eine ganz bedeutende Rolle im medizinischen Leben der Vereinigten Staaten und hat Grosses für die Kardiologie in Amerika geleistet».

Der Münchener Physiologe Otto Frank (1865-1944), ein bekannter Schüler Carl Ludwigs, nach dem das Frank-Starling-Gesetz mitbenannt ist,

42 Rudolf Thauer war noch 1944 Ordinarius am Physiologischen Institut in Danzig geworden, von 1951 bis zu seiner Emeritierung 1974 war er Direktor des Physiologischen Instituts der Justus-Liebig-Universität Giessen. Vgl. Kürschners Deutscher Gelehrten-Kalender 1987, 15. Ausgabe, Berlin/New York 1987, 4674 und 5346. In einem wenig günstigen Licht erscheint Thauer in den Lebenserinnerungen von Werner Schmidt: Leben an Grenzen. Autobiographischer Bericht eines Mediziners aus dunkler Zeit, Suhrkamp Taschenbuch Verlag, Frankfurt am Main 1993, 275.

43 Vgl. Anm. 40.

44 Vgl. Peter Kröner: Vor fünfzig Jahren. Die Emigration deutschsprachiger Wissenschaftler 1933-1939, Gesellschaft für Wissenschaftsgeschichte Münster 1983, 64. Zur Biographie von Kisch siehe auch Heinrich Jentgens, Bruno Kisch, Deutsche Medizinische Wochenschrift 91 (1966) 1853-1854. 
war der wissenschaftliche Mentor von Karl J. Wiggers (1883-1963). Nach seiner Ausbildung im Institut von Frank vor dem Ersten Weltkrieg wurde Wiggers Leiter des Physiologischen Instituts an der Western Reserve University in Cleveland. Ab 1930 galt er als der Stammvater der amerikanischen Kreislaufphysiologie, von dem sich die Mehrzahl der heutigen kardiovaskulären Physiologen in den USA ableitet ${ }^{45}$.

Die Anfänge der amerikanischen Gastroenterologie wurden durch Carl Anton Ewald (1845-1915) in Berlin entscheidend beeinflusst. Ismar Boas (1858-1938), einer seiner Schüler, liess sich 1886 in Berlin als erster Facharzt für Gastroenterologie nieder. 1895 begründete er die Zeitschrift Boas 'Archiv, später als Gastroenterologia und heute als Digestion bekannt. Die Gründung der ersten Gastroenterologischen Fachgesellschaft, der American Gastroenterological Association von 1897, ist auf seinen Einfluss und den des in Deutschland ausgebildeten John C. Hemmeter (1863-1931), Professor für Physiologie und Magenerkrankungen in Baltimore, zurückzuführen. In Deutschland entstand eine entsprechende Gesellschaft erst 1914 unter der Leitung von Carl Anton Ewald.

Unter weiteren amerikanischen Gastroenterologen, die in Deutschland ausgebildet wurden, war der Ewald-Schüler Max Einhorn (1862-1953), der 1888 nach einem Forschungsaufenthalt in Deutschland an der New York Postgraduate Medical School den ersten Lehrstuhl für Gastroenterologie erhielt ${ }^{46}$. Der Gastroenterologe Rudolf Schindler (1888-1968), Assistenzarzt im Krankenhaus München-Schwabing, war wegweisend beteiligt an der Entwicklung der Gastroskopie ${ }^{47}$. Er veröffentlichte 1923 das erste Lehrbuch und Atlas der Gastroskopie ${ }^{48}$. Zahlreiche Gastroenterologen aus dem Ausland, insbesondere aus den USA, kamen nach München, um bei Schindler diese Technik zu erlernen. 1932 entwickelte er mit Hilfe des Technikers Georg Wolf das Wolf-Schindler-Gastroskop, dessen flexible optische Achse der Gastroskopie zum klinischen Durchbruch verhalf. Nach der Machtergreifung der Nationalsozialisten wurde Schindler als «Halbjude» in ein Konzentrationslager gebracht. Auf Druck befreundeter Kollegen aus den USA

45 Hans Schaefer: Heidelberg, Bundesrepublik Deutschland, Persönliche Mitteilung vom 30. April 1993.

46 Vgl. Leonard Hoenig und James D. Boyle: The Life and Death of Ismar Boas, J Clin Gastroenterol 10 (1988) 16-24. Siehe auch Joseph B. Kirsner, The Specialization of Gastroenterology in America, Gastroenterology 104 (1993) 6-11.

47 Siehe Audrey B. Davis: Rudolf Schindler's Role in the Development of Gastroscopy, Bulletin of the History of Medicine 46 (1972) 150-170. Martin E. Gordon und Joseph B. Kirsner: Rudolf Schindler, Pioneer Endoscopist. Glimpses of the Man and his Work, Gastroenterology 77 (1979) 354-361.

48 Vgl. Gustav Paumgarnter: 47. Tagung der DGVS - Ansprache des Präsidenten, Z Gastroenterol 30 (1992) 837-840. 
und durch den Einfluss seines Schwagers, der selbst Nationalsozialist war, wurde die Freilassung erreicht, und Schindler lehrte ab 1934 an der Universität Chicago. Dort veröffentlichte er das Lehrbuch Gastroscopy. The Endoscopic Study of Gastric Pathology. 1941 gründete er in Chicago den American Gastroscopic Club, den Vorläufer der American Society of Gastrointestinal Endoscopy. Schindler lehnte eine Berufung als Professor nach Chicago ab, ging nach Kalifornien, 1958 nach Brasilien und kehrte in den 1960er Jahren nach München zurück, wo er 1968 verstarb. Seine technischen Entwicklungen und klinischen Anwendungen der gastrointestinalen Endoskopie gaben dieser Methode den entscheidenden Impuls im angloamerikanischen Raum. Parallel dazu haben Norbert Henning (1896-1985) und die spätere «Erlanger Schule» dieses Gebiet in Deutschland entwickelt.

Der führende Hepatologe Hans Popper (1903-1988) hatte seine klinische Ausbildung bei Hans Eppinger (1879-1946) in Wien erhalten ${ }^{49}$. Die wichtigste Arbeit aus seiner Wiener Zeit war ein Beitrag zur Entwicklung des Kreatinin-Clearance-Tests. 1938 emigrierte Popper in die Vereinigten Staaten $^{50}$ und erhielt eine Forschungsposition am Cook County Hospital in Chicago. Eppinger hatte seinem Lieblingsschüler erlaubt, ein Fluoreszenzmikroskop mitzunehmen. Untersuchungen mit diesem Mikroskop führten ihn dann von der Nephrologie zur Leberpathologie. 1943 wurde Popper Direktor des Pathologischen Instituts am Cook County Hospital. Er war der treibende Geist hinter der Gründung der American Association for the Study of the Liver Diseases (AASLD), deren erste informelle Sitzung 1948 abgehalten wurde. In den späten 1940er Jahren traf er die englische Hepatologin Sheila Sherlock (geb. 1918) bei einem Besuch in Yale. Dies führte zur Gründung der International Association for the Study of the Liver mit Sherlock als Gründungspräsidentin und Popper als zweitem Präsidenten. Der Freiburger Internist und Klinikchef Ludwig Heilmeyer (1899-1969) machte Popper anlässlich der Verleihung des Ehrendoktortitels der Universität Wien mit Herbert Falk (geb. 1924) bekannt, dem Inhaber einer Pharmazeutischen Firma in Freiburg. Poppers Einfluss brachte Falk dazu, die «Falk-Leberwochen» zu sponsern. 1957 wurde Popper Leiter der Pathologie am Mount Sinai Hospital in New York und später der erste Dekan der gleichnamigen Medical School. Dort setzte er seine Forschungen zur Aufklärung der viralen sowie der toxischen Hepatitis fort. 1976 wurde er Mitglied der National Academy of Science.

49 Rudi Schmid und Steven Schenker: Hans Popper in memoriam. 1903-1988, Hepatology 9 (1989) 669-674.

50 Vgl. Peter Kröner: Vor fünfzig Jahren. Die Emigration deutschsprachiger Wissenschaftler 1933-1939, Gesellschaft für Wissenschaftsgeschichte 1983, 77. 
Schliesslich möchten wir noch den Hämatologen Paul Heller (geb. 1914) vorstellen, der im Sudentenland des damaligen Österreich-Ungarn geboren wurde und an der deutschsprachigen Universität in Prag studierte. Kurz nach einer sechsmonatigen Assistentenzeit bei Eppinger in Wien wurde Heller als Jude und aufgrund seiner Mitgliedschaft in einer sozialdemokratischen Studentenorganisation unmittelbar nach dem Einmarsch Hitlers in die Tschechoslowakei 1938 verhaftet $^{51} .1939$ kurzfristig freigelassen, verbrachte er die Zeit von August 1939 bis Mai 1945 in den Konzentrationslagern Buchenwald und Auschwitz ${ }^{52}$. Bei der Befreiung Buchenwalds durch die Amerikaner fiel er dem bekannten amerikanischen Hörfunkreporter Edward R. Murrow auf, der ihn interviewte ${ }^{53}$ und ihm durch die resultierende Publicity half, in die Vereinigten Staaten zu gelangen. Nach Aufenthalten in New York, Omaha und Washington kam er 1954 an das Veterans Administration Hospital in Chicago. 1963 wurde Heller zum ordentlichen Professor des University of Illinois College of Medicine ernannt. Er war dort bis 1978 Leiter der Hämatologie und Onkologie. Seine Forschungen lagen auf dem Gebiet der abnormen Hämoglobine. Heller erinnert sich daran, dass es 1937 noch Gruppen amerikanischer Ärzte gab, die zu Weiterbildungskursen nach Wien kamen und dass deren geselliger Treffpunkt in Wien das Café Edison war.

Von den Ärzten, die während der Zeit des Nationalsozialismus aus deutschsprachigen Ländern in die Vereinigten Staaten oder andere Länder auswanderten, kehrten nur wenige nach dem Krieg in ihre Ursprungsländer zurück $^{54}$. Vielen der bereits etablierten Wissenschaftler und Professoren gelang es, in ihrem Immigrationsland eine angemessene Stellung zu erlangen: Die Hälfte der ordentlichen Professoren erreichte eine vergleichbare akademische Position, immerhin ein knappes Viertel der Extraordinarien. Dies gelang den ausserplanmässigen Professoren und Privatdozenten meist nicht; für 70\% dieser Gruppe bedeutete die Emigration das Ende ihrer akademischen Tätigkeit ${ }^{55}$. Ab 1937 wurde es für ausländische Ärzte immer schwieriger, in den USA approbiert zu werden. Die organisierte Ärzteschaft insbe-

51 Paul Heller: Chicago, IL, Persönliche Mitteilung vom 28. April 1993.

52 Vgl. Paul Heller: A Concentration Camp Diary. Holocaust Testimonies. Midstream, April 1980,29-36.

53 Vgl.Edward R. Murrow: In Search of Light. The Broadcasts of Edward R. Murrow 1938-1961, Alfred A. Knopf, New York 1967, 90-97.

54 Siehe Hans-Peter Kröner: Die Emigration deutschsprachiger Mediziner im Nationalsozialismus, Berichte zur Wissenschaftsgeschichte, Organ der Gesellschaft für Wissenschaftsgeschichte, Sonderheft, Weinheim 1989, 28. Von 3290 erfassten Emigranten kehrten lediglich $171(5,2 \%)$ nach dem Krieg in eines der Ursprungsländer (einschliesslich der Schweiz) zurück.

55 Siehe Hans-Peter Kröner: Die Emigration deutschsprachiger Mediziner im Nationalsozialismus, Berichte zur Wissenschaftsgeschichte, Organ der Gesellschaft für Wissenschaftsgeschichte, Sonderheft, Weinheim 1989, 21. 
sondere des Bundesstaates New York wehrte sich aus ökonomischen Gründen gegen die europäischen Einwanderer. Nach 1942 versagte das National Board of Medical Examiners Absolventen kontinentaleuropäischer, schottischer und irischer Fakultäten den Zugang zu den medizinischen Prüfungen. Erst wenn ein ausländischer Mediziner die amerikanische Staatsbürgerschaft erworben hatte, konnte er sich zur Prüfung anmelden ${ }^{56}$.

\section{Die Zeit von 1945 bis 1990}

Im Jahre 1945 war Deutschland physisch und moralisch zerstört, die USA jedoch auf einem bisher nicht erreichten wirtschaftlichen, politischen und auch wissenschaftlichen Höhepunkt. Die Medizin der USA übernahm unbestritten die Führungsrolle in der Welt. Diese Entwicklung war entscheidend beeinflusst durch die zentrale Förderung der biomedizinischen Forschung durch die staatlichen National Institutes of Health (NIH). 1887 war das National Institute of Hygiene gegründet worden - eine «zerbrechliche Verpflanzung vom Kontinent», speziell orientiert am Berliner Institut Robert Kochs. Basierend auf einer Empfehlung des Physikers Vannevar Bush (1890-1974) an Präsident Harry S. Truman (1884-1972) erstanden die NIH in ihrer heutigen Form in den ersten Nachkriegsjahren ${ }^{57}$.

Bei der Quellensuche stiessen wir auf bislang wenig bekannte Ereignisse, die uns James J. Smith (geb. 1914), emeritierter Ordinarius für Physiologie am Medical College of Wisconsin, ehemals Marquette University, mitteilte ${ }^{58}$. Smith war während des Zweiten Weltkriegs von 1942 bis 1945 in England als Mitglied einer Nachrichten-Sondereinheit der US-Luftwaffe eingesetzt. Waren Wernher von Braun (1912-1977) und seine Mitarbeiter nach dem Krieg als Raketentechniker in die USA gebracht worden, so bestand zwischen 1945 und 1947 die Aufgabe, deutsche biomedizinische Wissenschaftler in die USA zu bringen, um sich ihrer Mitarbeit in der Luft- und späteren Raumfahrtmedizin zu versichern. Die beiden prominentesten Wissenschaftler, die durch diese Operation Paper Clip («Unternehmen Büroklammer») in die USA kamen, waren Ulrich C. Luft (geb. 1910) und Otto H. Gauer (1909-1979). Der gebürtige Berliner Ulrich Luft war Experte in der Flugmedizin, sein Spezialgebiet die Höhenphysiologie. In den USA schrieb er ein Buch zur Höhenakklimatisation. Er arbeitete von 1947 bis 1953 an der US

$56 \mathrm{Vgl}$. Anm. 28.

57 Vgl. James B. Wyngaarden: Certain Aspects of Medical Research and its Support, Reviews of Infectious Diseases 9 (1987) Suppl. 5, 443-449.

58 James J. Smith: Milwaukee, WI, Persönliche Mitteilung vom 18. März 1993. 
Air Force School of Aviation Medicine in San Antonio, Texas, und leitete von 1954 bis 1980 das Department of Physiology der Lovelace Foundation in Albuquerque, New Mexico ${ }^{59}$. Der gebürtige Heidelberger Otto H. Gauer hatte nach seiner 1936 an der Ruperto Carola erfolgten Promotion seit 1937 am Luftfahrtmedizinischen Forschungsinstitut in Berlin gearbeitet. Er beschäftigte sich während des Krieges in Berlin und anschliessend von 1947 bis 1953 am Aeromedical Laboratory der Wright Field Airforce Base in Ohio mit Beschleunigungsphysiologie in der Luft- und Raumfahrttechnik. 1953 wechselte er zur Duke University in North Carolina, bevor er 1954 auf Betreiben von Rudolf Thauer nach Deutschland zurückkehrte und 1956 offiziell als Wissenschaftliches Mitglied an das Max-Planck-Institut für Physiologische und Klinische Forschung (W. G. Kerckhoff-Institut) nach Bad Nauheim berufen wurde. 1961 erhielt Gauer den Physiologischen Lehrstuhl an der Freien Universität Berlin ${ }^{60}$.

Weitere deutsche Physiologen, die nach dem Krieg in die USA gingen, waren Kurt Kramer (1906-1985), der von 1946 bis 1955 in der Flugmedizinischen Forschung arbeitete, bevor er nach Deutschland an den Göttinger Lehrstuhl für Physiologie zurückkehrte ${ }^{61}$. Ebenfalls aus Deutschland kam Theodor Benzinger, der zunächst am Bioenergetics Labortory des Naval Medical Research Institute in Bethesda, Maryland, arbeitete. Dort hat er 1949 die Methode der direkten Kalorimetrie veröffentlicht und die Thermoregulation des Menschen untersucht. Danach war er am National Bureau of Standards, Temperature Measurement and Standard Division in Washington tätig. Er lehnte 1959 den Ruf der Max-Planck-Gesellschaft an ein für ihn neu zu gründendes Institut in der Bundesrepublik Deutschland $\mathrm{ab}^{62}$.

Die wissenschaftliche Kontaktaufnahme nach 1945 begann zunächst durch informelle persönliche Begegnungen führender deutscher Kliniker und Theoretiker mit ihren angloamerikanischen Kollegen. Wie sich der Gastroenterologe und emeritierte Ordinarius für Innere Medizin an der Universität Erlangen-Nürnberg Ludwig Demling (geb. 1921) erinnert, waren die ersten Eindrücke zunächst unerwartet positiv und hilfreich ${ }^{63}$. In den 1950er

59 Ulrich Luft war seit 1938 Lehrbeauftragter und Luftfahrtmedizinischer Leiter der Abteilung Höhenphysiologie des Luftfahrtmedizinischen Forschungsinstituts in Berlin gewesen; 1943 habilitierte er sich. 1946/47 war er Kommissarischer Direktor des Physiologischen Instituts der Friedrich-Wilhelms-Universität. Vgl. Kürschners Deutscher Gelehrten-Kalender 1992, 16. Ausgabe, Berlin/New York 1992, 2252-2253.

$60 \mathrm{Vgl}$. Rudolf Thauer: Nachruf Otto H. Gauer, Max-Planck-Gesellschaft, Berichte und Mitteilungen, Sonderheft 1979.

61 Hans Schaefer: Heidelberg, Bundesrepublik Deutschland, Persönliche Mitteillung vom 30. April 1993.

62 Siehe Anm. 61.

63 Ludwig Demling: Schlüsselfeld, Bundesrepublik Deutschland, Persönliche Mitteilung vom 2. April 1993. 
Jahren begannen Studienreisen deutscher Hochschullehrer in die USA. So reisten im Auftrag des Westdeutschen Medizinischen Fakultätentages (WMFT) der Lehrstuhlinhaber für Physiologie in Heidelberg, Hans Schaefer (geb. 1906), sowie Rudolf Schoen (1892-1979), Direktor der Medizinischen Klinik in Göttingen, im Frühjahr 1953 auf Einladung der RockefellerFoundation in die USA, um amerikanische Lehrmethoden kennenzulernen und um später die deutschen Universitätspraktika reformieren zu können.Es kam mit Unterstützung der Fulbright-Stiftung aber schon bald auch wieder zu Gastprofessuren amerikanischer Wissenschaftler und Ärzte in Deutschland. In einer Umkehr der unmittelbaren Nachkriegssituation wurde nun James J. Smith nach seiner Teilnahme an der Operation Paper Clip von Hans Schaefer auf eine Fulbright-Gastprofessur nach Heildelberg eingeladen ${ }^{64}$.

Quantitativ bedeutsamer als die vereinzelte Rückkehr ehemaliger deutscher Wissenschaftler nach Deutschland war die zunehmende Zahl deutscher Studenten und junger Ärzte, die sich in die USA begaben, um dort zu studieren oder ihre Ausbildung fortzusetzen. Viele jener deutschen Mediziner blieben in den Vereinigten Staaten, da die wirtschaftlichen wie wissenschaftlichen Voraussetzungen dort so viel besser waren als im Deutschland der 1950er und 1960er Jahre. Als Beispiele möchten wir zum einen den Virologen Friedrich Deinhardt (geb. 1926) nennen, der nach seiner Ausbildung in den USA Leiter des Max-von-Pettenkofer-Instituts für Hygiene und Medizinische Mikrobiologie in München wurde, zum anderen den Schweizer Rudi Schmid (geb. 1922), der nach seiner Ausbildung in Minneapolis Dekan der Medizinischen Fakultät an der University of California in San Francisco wurde ${ }^{65}$.

In den 1950er Jahren begannen die Deutsche Forschungsgemeinschaft, der Deutsche Akademische Austauschdienst, die Alexancer von Humboldt-Stiftung und die Max-Planck-Gesellschaft den internationalen wissenschaftlichen Austausch zu fördern. Diese Stiftungen hatten unterschiedliche Schwerpunkte, ihnen gemeinsam jedoch war es, deutsche Wissenschaftler im Ausland und ausländische Wissenschaftler in Deutschland zu unterstützen. Biomedizinische und internistische Forschung wurde vielfältig gefördert, und sehr viele deutsche Ärzte und Grundlagenforscher konnten auf diese Weise in den USA wie auch in Deutschland forschen oder ihre Ausbildung fortsetzen. Die Zahl der Amerikaner, die in Deutschland etwa mit Hilfe eines Humboldt-Stipendiums forschten, ist klein im Vergleich mit den vielen Hunder-

64 James J. Smith: Milwaukee, WI, Persönliche Mitteilung vom 18. März 1993.

65 Rudi Schmid: San Francisco, CA, Persönliche Mitteilung vom 8. März 1993.

66 Humboldt Awards for U.S. Scientists, in: Alexander von Humboldt Foundation 1953-1983, 187-190. (Deutsche Version: Humboldt-Preise für Naturwissenschaftler aus USA, in: Alex- 
ten deutscher Wissenschaftler, die in Amerika ausgebildet wurden. Rund ein Drittel aller deutschen Wissenschaftler, die mit Unterstützung der Alexander von Humboldt-Stiftung die USA besucht hatten, blieb auf Dauer im Gastland. Leider gab und gibt es keine medizinischen Forschungsprogramme, die gemeinsam von deutschen und amerikanischen Stiftungen gefördert werden ${ }^{66}$.

Wie vor dem Ersten Weltkrieg die deutschsprachige Medizin die amerikanische Forschung, Klinik und Ausbildung geprägt hatte, so beeinflusste im Gegenzug die amerikanische Innere Medizin nach dem Zweiten Weltkrieg die europäische Klinik. Seit Beginn der 1980er Jahre hat sich die deutschsprachige Innere Medizin allerdings wieder stärker von der amerikanischen Vormachtstellung emanzipiert. Deutschsprachige Wissenschaftler veröffentlichen zunehmend in den führenden amerikanischen Fachzeitschriften. So beträgt zum Beispiel der Anteil der Publikationen aus deutschsprachigen Ländern in der Fachzeitschrift Gastroenterology mittlerweile 5\%, nur durch Veröffentlichungen aus den USA selbst und aus Japan übertroffen ${ }^{67}$. Heute begeben sich junge deutsche oder schweizerische Internisten nicht mehr in die USA, um eine klinische Ausbildung zu erwerben; vielmehr gehen sie in die USA, um gezielt eine Ausbildung in Fächern der vorklinischen und klinischen Grundlagenforschung zu erwerben.

So waren und sind die wechselseitigen Einflüsse zwischen der deutschen und amerikanischen Inneren Medizin vielfältig, zeitspezifisch verschieden, jedoch ohne zentrale Planung: Die US-amerikanische Innere Medizin des späten 19. Jahrhunderts beruhte ganz wesentlich auf dem Vorbild der deutschen Medizin, eine Situation, die sich nach dem Ersten Weltkrieg grundlegend änderte. Nach dem Zweiten Weltkrieg übernahm die Medizin der USA die führende Rolle in der Welt. Amerikanische Forschung und Klinik wurden zum Modell für die westliche Medizin und somit auch für die deutschsprachigen Länder. Während der 1980er Jahre hat sich die deutschsprachige klinische Forschung jedoch wieder zu einer annähernd ebenbürtigen Partnerin der amerikanischen Inneren Medizin gewandelt ${ }^{68}$.

ander von Humboldt-Stiftung 1953-1983, Bonn 1984, 201-214.) Feodor Lynen Research Fellowships for German Academics, in: Alexander von Humboldt Foundation 1953-1983, 204-209. (Deutsche Version: Feodor-Lynen-Forschungsstipendien für deutsche Wissenschaftler, in: Alexander von Humdoldt-Stiftung 1953-1983, Bonn 1984, 218-224.) Heinrich Pfeiffer: Forword. The U.S. Special Program in the Past Ten Years. Report on the Sponsorship of Senior U.S. Scientists as Recipients of the Humboldt Award form 1972-1981, in: Alexander von Humboldt Foundation 1953-1983, 5-11.

67 Gastroenterology, Editorial Office: Rochester, MN, Persönliche Mitteilung vom 9. Juni 1993.

68 Zur gegenwärtigen Entwicklung vgl. Reinhard Lohölter: Zur internationalen Entwicklung der Studienreform in der Medizin, in: Dietrich Habeck; Udo Schagen und Günther Wagner (Hg.), Reform der Ärzteausbildung. Neue Wege in den Fakultäten, Blackwell Wissenschaft, Berlin 1993, 51-69. 